International Journal of Pure and Applied Mathematics

Volume 102 No. 3 2015, 547-561

ISSN: 1311-8080 (printed version); ISSN: 1314-3395 (on-line version)

url: http://www.ijpam.eu

doi: http://dx.doi.org/10.12732/ijpam.v102i3.12

ijpam.eu

\title{
COMMON FIXED POINTS FOR WEAKLY COMPATIBLE MAPPINGS SATISFYING IMPLICIT FUNCTIONS IN MULTIPLICATIVE METRIC SPACES
}

Chahn Yong Jung ${ }^{1}$, Parveen Kumar ${ }^{2}$, Sanjay Kumar ${ }^{3}$, Shin Min Kang ${ }^{4} \S$

${ }^{1}$ Department of Business Administration

Gyeongsang National University

Jinju 660-701, KOREA

${ }^{2,3}$ Department of Mathematics

Deenbandhu Chhotu Ram University of Science and Technology

Murthal, Sonipat 131039, Haryana, INDIA

${ }^{4}$ Department of Mathematics and RINS

Gyeongsang National University

Jinju 660-701, KOREA

\begin{abstract}
In this paper we prove common fixed point theorems for weakly compatible mapping in multiplicative metric spaces. Next, we prove common fixed point theorems for weakly compatible mappings along with E.A and common limit range properties.
\end{abstract}

AMS Subject Classification: 47H10, 54H 25

Key Words: multiplicative metric space, weakly compatible mapping, implicit function, E.A and common limit range properties

\section{Introduction and Preliminaries}

It is well know that the set of positive real numbers $\mathbb{R}_{+}$is not complete according

Received: May 21, 2015

(C) 2015 Academic Publications, Ltd.

$\S$ Correspondence author url: www.acadpubl.eu 
to the usual metric. To overcome this problem, in 2008, Bashirov et al. [4] introduced the concept of multiplicative metric spaces as follows:

Definition 1.1. Let $X$ be a nonempty set. A multiplicative metric is a mapping $d: X \times X \rightarrow \mathbb{R}_{+}$satisfying the following conditions:

(i) $d(x, y) \geq 1$ for all $x, y \in X$ and $d(x, y)=1$ if and only if $x=y$;

(ii) $d(x, y)=d(y, x)$ for all $x, y \in X$;

(iii) $d(x, y) \leq d(x, z) \cdot d(z, y)$ for all $x, y, z \in X$ (multiplicative triangle inequality).

Example 1.2. ([11]) Let $\mathbb{R}_{+}^{n}$ be the collection of all $n$-tuples of positive real numbers. Let $d: \mathbb{R}_{+}^{n} \times \mathbb{R}_{+}^{n} \rightarrow \mathbb{R}$ be defind as follows:

$$
d(x, y)=\left(\left|\frac{x_{1}}{y_{1}}\right| \cdot\left|\frac{x_{2}}{y_{2}}\right| \cdots\left|\frac{x_{n}}{y_{n}}\right|\right),
$$

where $x=\left(x_{1}, \ldots, x_{n}\right), y=\left(y_{1}, \ldots, y_{n}\right) \in \mathbb{R}_{+}^{n}$ and $|\cdot|: \mathbb{R}_{+} \rightarrow \mathbb{R}_{+}$is defined by

$$
|a|= \begin{cases}a & \text { if } a \geq 1 ; \\ \frac{1}{a} & \text { if } a<1 .\end{cases}
$$

Then it is obvious that all conditions of a multiplicative metric are satisfied.

Example 1.3. ([15]) Let $d: \mathbb{R} \times \mathbb{R} \rightarrow[1, \infty)$ be defined as $d(x, y)=a^{|x-y|}$, where $x, y \in \mathbb{R}$ and $a>1$. Then $d$ is a multiplicative metric.

Remark 1.4. We note that the Example 1.2 is valid for positive real numbers and Example 1.3 is valid for all real numbers.

One can refer to $[6,11]$ for detailed multiplicative metric topology.

Definition 1.5. Let $(X, d)$ be a multiplicative metric space. Then a sequence $\left\{x_{n}\right\}$ in $X$ said to be

(1) a multiplicative convergent to $x$ if for every multiplicative open ball $B_{\epsilon}(x)=\{y \mid d(x, y)<\epsilon\}, \epsilon>1$, there exists a natural number $N$ such that $n \geq N$, then $x_{n} \in B_{\epsilon}(x)$, that is, $d\left(x_{n}, x\right) \rightarrow 1$ as $n \rightarrow \infty$.

(2) a multiplicative Cauchy sequence if for all $\epsilon>1$, there exists $N \in \mathbb{N}$ such that $d\left(x_{n}, x_{m}\right)<\epsilon$ for all $m, n>N$, that is, $d\left(x_{n}, x_{m}\right) \rightarrow 1$ as $n \rightarrow \infty$.

(3) We call a multiplicative metric space complete if every multiplicative Cauchy sequence in it is multiplicative convergent to $x \in X$. 
Remark 1.6. The set of positive real numbers $\mathbb{R}_{+}$is not complete according to the usual metric. Let $X=\mathbb{R}_{+}$and the sequence $\left\{x_{n}\right\}=\left\{\frac{1}{n}\right\}$. It is obvious $\left\{x_{n}\right\}$ is a Cauchy sequence in $X$ with respect to usual metric and $X$ is not a complete metric space, since $0 \notin \mathbb{R}_{+}$. In case of a multiplicative metric space, we take a sequence $\left\{x_{n}\right\}=\left\{a^{\frac{1}{n}}\right\}$, where $a>1$. Then $\left\{x_{n}\right\}$ is a Cauchy sequence since for $n \geq m$,

$$
\begin{aligned}
d\left(x_{n}, x_{m}\right) & =\left|\frac{x_{n}}{x_{m}}\right|=\left|\frac{a^{\frac{1}{n}}}{a^{\frac{1}{m}}}\right|=\left|a^{\frac{1}{n}-\frac{1}{m}}\right| \\
& \leq a^{\frac{1}{m}-\frac{1}{n}}<a^{\frac{1}{m}}<\epsilon \quad \text { if } m>\frac{\log a}{\log \epsilon},
\end{aligned}
$$

where $|a|=\left\{\begin{array}{ll}a & \text { if } a \geq 1, \\ \frac{1}{a} & \text { if } a<1 .\end{array}\right.$ Also, $\left\{x_{n}\right\} \rightarrow 1$ as $n \rightarrow \infty$ and $1 \in \mathbb{R}_{+}$. Hence $(X, d)$ is a complete multiplicative metric space.

In 2012, Özavsar and Çevikel [11] gave the concept of multiplicative contraction mappings and proved some fixed point theorem of such mappings in a multiplicative metric space.

Definition 1.7. Let $f$ be a mapping of a multiplicative metric space $(X, d)$ into itself. Then $f$ is said to be a multiplicative contraction if there exists a real constant $\lambda \in[0,1)$ such that

$$
d(f x, f y) \leq d^{\lambda}(x, y) \quad \text { for all } x, y \in X .
$$

Gu et. al. [5] introduced the notion of commutative and weak commutative mappings in a multiplicative metric space and proved some fixed point theorems for these mappings.

Definition 1.8. Let $f$ and $g$ be two mappings of a multiplicative metric space $(X, d)$ into itself. Then $f$ and $g$ are said to be

(1) commutative mappings if $f g x=g f x$ for all $x \in X$.

(2) weak commutative mappings if $d(f g x, g f x) \leq d(f x, g x)$ for all $x \in X$.

Notice that commuting mappings are obviously weakly commuting. However, the converse need not be true. 
Example 1.9. Let $X=[0,1]$ be a multiplicative metric $d$ on $X$ defined by $d(x, y)=a^{|x-y|}$, where for all $x, y \in X$ and $a>1$. Define mappings $f$ and $g: X \rightarrow X$ by $f x=\frac{x}{3-x}$ and $g x=\frac{x}{3}$ for all $x \in X$. For any $x \in X$,

$$
d(f g x, g f x)=a^{\left|\frac{2 x^{2}}{(9-x)(9-3 x)}\right|} \leq a^{\left|\frac{x^{2}}{9-3 x}\right|}=d(f x, g x) .
$$

Then $f$ and $g$ are weakly commuting but $f$ and $g$ are not commuting since

$$
f g x=\frac{x}{9-x}<\frac{x}{9-3 x}=g f x
$$

for any non-zero $x \in X$.

In metric spaces, they introduced the notions of weak compatibility $[9,10]$, E.A. property [1] and common limit range property [8, 17].

Now, we introduce the notions in multiplicative metric spaces

Definition 1.10. Let $f$ and $g$ be two mappings of a multiplicative metric space $(X, d)$ into itself. Then $f$ and $g$ are said to be weakly compatible if they commute at coincidence points, that is, if $f t=g t$ for some $t \in X$ implies that $f g t=g f t$.

Notice that weakly commuting mappings are obviously weakly compatible. However, the converse need not be true.

Example 1.11. Let $X=[0, \infty)$ be a multiplicative metric $d$ on $X$ defined by $d(x, y)=a^{|x-y|}$, where for all $x, y \in X$ and $a>1$. Define mappings $f$ and $g: X \rightarrow X$ by $f x=x^{2}$ and $g x=2 x^{2}$ for all $x \in X$. So we have $f g x=4 x^{4}$ and gf $x=2 x^{4}$ for all $x \in X$. For any $x \in X$,

$$
d(f g x, g f x)=a^{2 x^{4}} \not \leq a^{x^{2}}=d(f x, g x) .
$$

Then $f$ and $g$ are not weakly commuting but $f$ and $g$ are weakly compatible since $f 0=g 0$ for some $0 \in X$ implies $f g 0=g f 0$.

Definition 1.12. Let $f$ and $g$ be two mappings of a multiplicative metric space $(X, d)$ into itself. Then $f$ and $g$ are said to satisfy E.A property if there exists a sequence $\left\{x_{n}\right\}$ in $X$ such that $\lim _{n \rightarrow \infty} f x_{n}=\lim _{n \rightarrow \infty} g x_{n}=t$ for some $t \in X$. 
Example 1.13. Let $X=[1, \infty)$. Define $d: X^{2} \rightarrow R_{+}$by $d(x, y)=a^{|x-y|}$, $a>1$. Then $(X, d)$ be a multiplicative metric space. Define $f, g: X \rightarrow X$ as $f x=\frac{1}{x}$ and $g x=\frac{1}{x^{2}}$. Consider the sequence $\left\{x_{n}\right\}=\left\{a^{1 / n}\right\}, a>1$ in $X$. Now $\lim _{n \rightarrow \infty} f x_{n}=\lim _{n \rightarrow \infty} g x_{n}=1 \in X$. Hence $f$ and $g$ satisfies E.A. property.

Definition 1.14. Let $f$ and $g$ be two mappings of a multiplicative metric space $(X, d)$ into itself. Then $f$ and $g$ are said to satisfy $C L R_{g}$ property (common limit range of $g$ property) if there exists a sequence $\left\{x_{n}\right\}$ in $X$ such that $\lim _{n \rightarrow \infty} f x_{n}=\lim _{n \rightarrow \infty} g x_{n}=g t$ for some $t \in X$.

Example 1.15. Let $X=[1, \infty)$. Define $d: X^{2} \rightarrow R_{+}$by $d(x, y)=a^{\mid} x-y \mid$, $a>1$. Then $(X, d)$ be a multiplicative metric space. Define $f, g: X \rightarrow X$ as $f x=x^{2}$ and $g x=x^{3}$. Consider the sequence $\left\{x_{n}\right\}=\left\{a^{1 / n}\right\}, a>1$ in $X$. Now $\lim _{n \rightarrow \infty} f x_{n}=\lim _{n \rightarrow \infty} g x_{n}=1=g 1$ and $1 \in X$. Hence $f$ and $g$ satisfies $C L R_{g}$ property.

Definition 1.16. Let $f, g$ and $h, k$ be mappings of a multiplicative metric space $(X, d)$ into itself. Then the pairs $f, g$ and $h, k$ are said to share common limit in the range of $g$ property if there exist sequences $\left\{x_{n}\right\}$ and $\left\{y_{n}\right\}$ in $X$ such that $\lim _{n \rightarrow \infty} f x_{n}=\lim _{n \rightarrow \infty} g x_{n}=\lim _{n \rightarrow \infty} h y_{n}=\lim _{n \rightarrow \infty} k y_{n}=g t$ for some $t \in X$.

\section{Main Results}

Recently, Popa [12] used the implicit function rather than contraction conditions to prove fixed point theorems in metric spaces. The strength of implicit relation unifies several contraction conditions at the same time. This fact is seen from examples furnished in Popa [12]. Implicit relations on metric spaces have been used by many authors (for details see $[2,3,7,13,14,16,18]$ and their references therein).

In this section, we define a suitable class of the implicit function involving five real non-negative arguments as follows:

Let $\Phi$ denote the family of functions such that $\phi: \mathbb{R}_{+}^{5} \rightarrow \mathbb{R}_{+}$is continuous and increasing in each coordinate variable and $\phi(t, 1,1, t, t) \leq t, \phi(1, t, 1, t, 1) \leq$ $t, \phi(1,1, t, 1, t) \leq t, \phi\left(t_{1}, t_{1}, t, 1, t_{1} t\right) \leq t_{1} t, \phi\left(t_{1}, t, t_{1}, t_{1} t, 1\right) \leq t_{1} t$ for every $t, t_{1} \in \mathbb{R}_{+}\left(t, t_{1} \geq 1\right)$.

Obviously $\phi(1,1,1,1,1)=1$. 
There exists many functions $\phi$ which belongs to $\Phi$ :

Example 2.1. Let $\phi: \mathbb{R}_{+}^{5} \rightarrow \mathbb{R}_{+}$be defined by

$$
\phi\left(t_{1}, t_{2}, t_{3}, t_{4}, t_{5}\right)=t_{1}+t_{2}+t_{3}-t_{4}-t_{5},
$$

then $\phi \in \Phi$.

Example 2.2. Let $\phi: \mathbb{R}_{+}^{5} \rightarrow \mathbb{R}^{+}$be defined by

$$
\phi\left(t_{1}, t_{2}, t_{3}, t_{4}, t_{5}\right)=t_{1}+1-\max \left\{t_{2}, t_{3}, t_{4}, t_{5}\right\},
$$

then $\phi \in \Phi$.

Example 2.3. Let $\phi: \mathbb{R}_{+}^{5} \rightarrow \mathbb{R}^{+}$be defined by

$$
\phi\left(t_{1}, t_{2}, t_{3}, t_{4}, t_{5}\right)=\max \left\{t_{1}, t_{2}, t_{3}, t_{4}, t_{5}\right\}
$$

then $\phi \in \Phi$.

Example 2.4. Let $\phi: \mathbb{R}_{+}^{5} \rightarrow \mathbb{R}^{+}$be defined by

$$
\phi\left(t_{1}, t_{2}, t_{3}, t_{4}, t_{5}\right)=\left[\max \left\{t_{1}, t_{2}, t_{3}, t_{4}, t_{5}\right\}\right]^{1 / 2},
$$

then $\phi \in \Phi$.

Now we prove the following theorems for weakly compatible mappings satisfying the implicit function in a multiplicative metric space as follow:

Theorem 2.5. Let $A, B, S$ and $T$ be mappings of a multiplicative metric space $(X, d)$ into itself satisfying $S X \subset B X, \quad T X \subset A X$

$$
\begin{aligned}
d(S x, T y) \leq[\phi(d(A x, B y), d(A x, S x), & \\
& d(B y, T y), d(S x, B y), d(A x, T y)\}]^{\lambda}
\end{aligned}
$$

for all $x, y \in X$, where $\lambda \in\left(0, \frac{1}{2}\right)$ and $\phi \in \Phi$;

(C3) Assume that the pairs $A, S$ and $B, T$ are weakly compatible;

(C4) One of the subspace $A X$ or $B X$ or $S X$ or $T X$ is complete.

Then $A, B, S$ and $T$ have a unique common fixed point. 
Proof. Let $x_{0} \in X$ be an arbitrary point. Since $S X \subset B X$, there exists $x_{1} \in X$ such that $S x_{0}=B x_{1}=y_{0}$. Now for this $x_{1}$ there exists $x_{2} \in X$ such that $T x_{1}=A x_{2}=y_{1}$. Similarly, we can inductively define a sequence $\left\{y_{n}\right\}$ such that

$$
S x_{2 n}=B x_{2 n+1}=y_{2 n}, \quad T x_{2 n+1}=A x_{2 n+2}=y_{2 n+1} .
$$

From $(C 2)$, we have

$$
\begin{aligned}
& d\left(y_{2 n}, y_{2 n+1}\right) \\
&= d\left(S x_{2 n}, T x_{2 n+1}\right) \\
& \leq {\left[\phi \left(d\left(A x_{2 n}, B x_{2 n+1}\right), d\left(A x_{2 n}, S x_{2 n}\right),\right.\right.} \\
&\left.\left.d\left(B x_{2 n+1}, T x_{2 n+1}\right), d\left(S x_{2 n}, B x_{2 n+1}\right), d\left(A x_{2 n}, T x_{2 n+1}\right)\right\}\right]^{\lambda} \\
&= {\left[\phi \left(d\left(y_{2 n-1}, y_{2 n}\right), d\left(y_{2 n-1}, y_{2 n}\right),\right.\right.} \\
&\left.\left.\quad d\left(y_{2 n}, y_{2 n+1}\right), d\left(y_{2 n}, y_{2 n}\right), d\left(y_{2 n-1}, y_{2 n+1}\right)\right\}\right]^{\lambda} \\
& \leq {\left[\phi \left(d\left(y_{2 n-1}, y_{2 n}\right), d\left(y_{2 n-1}, y_{2 n}\right),\right.\right.} \\
&\left.\left.\quad d\left(y_{2 n}, y_{2 n+1}\right), 1, d\left(y_{2 n-1}, y_{2 n}\right) \cdot d\left(y_{2 n}, y_{2 n+1}\right)\right\}\right]^{\lambda} \\
& \leq d^{\lambda}\left(y_{2 n-1}, y_{2 n}\right) \cdot d^{\lambda}\left(y_{2 n}, y_{2 n+1}\right) .
\end{aligned}
$$

This implies that $d\left(y_{2 n}, y_{2 n+1}\right) \leq d^{\frac{\lambda}{1-\lambda}}\left(y_{2 n-1}, y_{2 n}\right)$. On putting $\frac{\lambda}{1-\lambda}=h$.

$$
d\left(y_{2 n}, y_{2 n+1}\right) \leq d^{h}\left(y_{2 n-1}, y_{2 n}\right)
$$

Similarly we obtain

$$
d\left(y_{2 n+1}, y_{2 n+2}\right) \leq d^{h}\left(y_{2 n}, y_{2 n+1}\right)
$$

Hence

$$
d\left(y_{n}, y_{n+1}\right) \leq d^{h}\left(y_{n-1}, y_{n}\right) \leq d^{h^{2}}\left(y_{n-2}, y_{n-1}\right) \leq \cdots \leq d^{h^{n}}\left(y_{0}, y_{1}\right)
$$

for all $n \geq 2$. Let $m, n \in \mathbb{N}$ such that $m \geq n$. Then we get

$$
\begin{aligned}
d\left(y_{m}, y_{n}\right) & \leq d\left(y_{m}, y_{m-1}\right) \cdot d\left(y_{m-1}, y_{m-2}\right) \cdots d\left(y_{n+1}, y_{n}\right) \\
& \leq d^{h^{m-1}}\left(y_{1}, y_{0}\right) \cdot d^{h^{m-2}}\left(y_{1}, y_{0}\right) \cdots d^{h^{n}}\left(y_{1}, y_{0}\right) \\
& \leq d^{\frac{h^{n}}{1-h}}\left(y_{1}, y_{0}\right) .
\end{aligned}
$$

Letting limit as $m, n \rightarrow \infty$, we have $d\left(y_{m}, y_{n}\right) \rightarrow 1$. Therefore $\left\{y_{n}\right\}$ is a multiplicative Cauchy sequence. 
Now, suppose that $A X$ is complete there exists $u \in A X$ such that

$$
y_{2 n+1}=T x_{2 n+1}=A x_{2 n+2} \rightarrow u \quad \text { as } n \rightarrow \infty .
$$

Consequently, we can find $v \in X$ such that $A v=u$. Further a multiplicative Cauchy sequence $\left\{y_{n}\right\}$ has a convergent subsequence $\left\{y_{2 n+1}\right\}$, therefore, the sequence $\left\{y_{n}\right\}$ converges and hence a subsequence $\left\{y_{2 n}\right\}$ also converges. Thus we have

$$
y_{2 n}=S x_{2 n}=B x_{2 n+1} \rightarrow u \quad \text { as } n \rightarrow \infty .
$$

We claim $S v=u$. Putting $x=v$ and $y=x_{2 n+1}$ in $(C 2)$, we get

$$
\begin{aligned}
d\left(S v, y_{2 n+1}\right)= & d\left(S v, T x_{2 n+1}\right) \\
\leq & {\left[\phi \left(d\left(A v, B x_{2 n+1}\right), d(A v, S v)\right.\right.} \\
& \left.\left.\quad d\left(B x_{2 n+1}, T x_{2 n+1}\right), d\left(S v, B x_{2 n+1}\right), d\left(A v, T x_{2 n+1}\right)\right)\right]^{\lambda} .
\end{aligned}
$$

Letting $n \rightarrow \infty$, we have

$$
d(S v, u) \leq[\phi(1, d(u, S v), 1, d(S v, u), 1)]^{\lambda} \leq d^{\lambda}(S v, u)
$$

this implies that $d(S v, u)=1$ and hence $u=S v$. Since $u=S v \in S X \subset B X$ there exists $w \in X$ such that $u=B w$.

We claim $T w=u$. Putting $x=v$ and $y=w$ in $(C 2)$, we have

$$
\begin{aligned}
d(u, T w)= & d(S v, T w) \\
\leq & {[\phi(d(A v, B w), d(A v, S v)} \\
& d(B w, T w), d(S v, B w), d(A v, T w))]^{\lambda} \\
= & {[\phi(1,1, d(u, T w), 1, d(u, T w))]^{\lambda} } \\
\leq & d^{\lambda}(u, T w)
\end{aligned}
$$

this implies that $d(u, T w)=1$ and hence $u=T w$. Hence we get $u=A v=S v$, that is, $v$ is a coincidence point of $A$ and $S$ and $u=B w=T w$, that is $w$ is a coincidence point of $B$ and $T$. Therefore $A v=S v=B w=T w=u$.

Since the pairs $A, S$ and $B, T$ are weakly compatible, we have

$$
S u=S(A v)=A(S v)=A u=w_{1}(\text { say })
$$

and

$$
T u=T(B w)=B(T w)=B u=w_{2}(\text { say })
$$


From $(C 2)$, we have

$$
\begin{aligned}
d\left(w_{1}, w_{2}\right)= & d(S u, T u) \\
\leq & {[\phi(d(A u, B u), d(A u, S u),} \\
& d(B u, T u), d(S u, B u), d(A u, T u))]^{\lambda} \\
= & {\left[\phi\left(d\left(w_{1}, w_{2}\right), 1,1, d\left(w_{1}, w_{2}\right), d\left(w_{1}, w_{2}\right)\right)\right]^{\lambda} } \\
\leq & d^{\lambda}\left(w_{1}, w_{2}\right),
\end{aligned}
$$

this implies that $w_{1}=w_{2}$. and hence we have $S u=A u=T u=B u$.

Again using $(C 2)$, we have

$$
\begin{aligned}
d(S v, T u) \leq & {[\phi(d(A v, B u), d(A v, S v),} \\
& d(B u, T u), d(S v, B u), d(A v, T u))]^{\lambda} \\
= & {[\phi(d(S v, T u), 1,1, d(S v, T u), d(S v, T u))]^{\lambda} } \\
\leq & d^{\lambda}(S v, T u),
\end{aligned}
$$

this implies that $S v=T u(u=T u)$ and hence we have $u=S u=A u=T u=$ $B u$. Therefore $u$ is a common fixed point of $A, B, S$ and $T$.

Similarly, we can complete the proof for cases in which $B X$ or $T X$ or $S X$ is complete.

The uniqueness can be easily follows from $(C 2)$. This completes the proof.

In Theorem 2.3. if we put $S=T$, then we obtains the following corollary.

Corollary 2.6. Let $A, B$ and $S$ be mappings of a multiplicative metric space $(X, d)$ into itself satisfying

$$
\begin{gathered}
S X \subset B X, \quad S X \subset A X ; \\
d(S x, S y) \leq[\phi(d(A x, B y), d(A x, S x), \\
d(B y, S y), d(S x, B y), d(A x, S y)\}]^{\lambda}
\end{gathered}
$$

for all $x, y \in X$, where $\lambda \in\left(0, \frac{1}{2}\right)$ and $\phi \in \Phi$;

(c3) the pairs $A, S$ and $B, S$ are weakly compatible;

(c4) one of the subspace $A X$ or $B X$ or $S X$ is complete.

Then $A, B$ and $S$ have a unique common fixed point. 
In Theorem 2.3, if we put $A=B=I$, then we obtains the corollary.

Corollary 2.7. Let $S$ and $T$ be mappings of a multiplicative metric space $(X, d)$ into itself satisfying

$$
\begin{aligned}
d(S x, T y) \leq[\phi(d(x, y), d(x, S x) & \\
& d(y, T y), d(S x, y), d(x, T y))]^{\lambda}
\end{aligned}
$$

for all $x, y \in X$, where $\lambda \in\left(0, \frac{1}{2}\right)$ and $\phi \in \Phi$;

(c6) one of the subspace $S X$ or $T X$ is complete.

Then $S$ and $T$ have a unique common fixed point.

Next we prove the following theorems for weakly compatible mappings with E.A. property satisfying the implicit function in a multiplicative metric space as follow:

Theorem 2.8. Let $A, B, S$ and $T$ be mappings of a multiplicative metric space $(X, d)$ into itself satisfying the conditions $(C 1),(C 2),(C 3)$ and the following conditions:

(C5) One of the subspace $A X$ or $B X$ or $S X$ or $T X$ is closed subset of $X$;

(C6) the pairs $A, S$ and $B, T$ satisfies the E.A. property.

Then $A, B, S$ and $T$ have a unique common fixed point.

Proof. Suppose that the pair $A, S$ satisfies the E.A property. Then there exists a sequence $\left\{x_{n}\right\}$ in $X$ such that $\lim _{n \rightarrow \infty} A x_{n}=\lim _{n \rightarrow \infty} S x_{n}=z$ for some $z \in X$. Since $S X \subset B X$, there exists a sequence $\left\{y_{n}\right\}$ in $X$ such that $S x_{n}=B y_{n}$. Hence $\lim _{n \rightarrow \infty} B y_{n}=z$.

Now, suppose that $B X$ is a closed subset of $X$, there exists a point $u \in X$ such that $B u=z$.

We will show that $\lim _{n \rightarrow \infty} T y_{n}=z$. From inequality $(C 2)$, we have

$$
\begin{aligned}
d\left(S x_{n}, T y_{n}\right) \leq[ & \phi\left(d\left(A x_{n}, B y_{n}\right), d\left(A x_{n}, S x_{n}\right)\right. \\
& \left.\left.d\left(B y_{n}, T y_{n}\right), d\left(S x_{n}, B y_{n}\right), d\left(A x_{n}, T y_{n}\right)\right)\right]^{\lambda}
\end{aligned}
$$

Letting $n \rightarrow \infty$, we have

$$
\begin{aligned}
d\left(z, T y_{n}\right) & \leq\left[\phi\left(1,1, d\left(z, T y_{n}\right), 1, d\left(z, T y_{n}\right)\right)\right]^{\lambda} \\
& \leq d^{\lambda}\left(z, T y_{n}\right)
\end{aligned}
$$


which implies that $\lim _{n \rightarrow \infty} d\left(z, T y_{n}\right)=1$. Thus we have

$$
\lim _{n \rightarrow \infty} A x_{n}=\lim _{n \rightarrow \infty} B y_{n}=\lim _{n \rightarrow \infty} S x_{n}=\lim _{n \rightarrow \infty} T y_{n}=z=B u
$$

for some $u \in X$.

Putting $x=x_{n}$ and $y=u$ in $(C 2)$, we have

$$
\begin{aligned}
d\left(S x_{n}, T u\right) \leq[ & \phi\left(d\left(A x_{n}, B u\right), d\left(A x_{n}, S x_{n}\right),\right. \\
& \left.\left.d(B u, T u), d\left(S x_{n}, B u\right), d\left(A x_{n}, T u\right)\right)\right]^{\lambda} .
\end{aligned}
$$

Letting $n \rightarrow \infty$, we have

$$
\begin{aligned}
d(B u, T u) & \leq[\phi(1,1, d(B u, T u), 1, d(B u, T u))]^{\lambda} \\
& \leq d^{\lambda}(B u, T u),
\end{aligned}
$$

which implies that $B u=T u$. Since the pair $B, T$ is weakly compatible, we have $B T u=T B u$ and then $B B u=B T u=T B u=T T u$.

On the other way, since $T X \subset A X$, there exists $v \in X$ such that $T u=A v$.

Next we claim that $A v=S v$. Putting $x=v$ and $y=u$, we have

$$
\begin{aligned}
d(S v, T u) \leq & {[\phi(d(A v, B u), d(A v, S v),} \\
& d(B u, T u), d(S v, B u), d(A v, T u))]^{\lambda} .
\end{aligned}
$$

Letting $n \rightarrow \infty$, we have

$$
\begin{aligned}
d(S v, A v) & \leq[\phi(1, d(A v, S v), 1, d(A v, S v), 1)]^{\lambda} \\
& \leq d^{\lambda}(S v, A v),
\end{aligned}
$$

which implies that $S v=A v$ and hence $B u=T u=A v=S v$. Since the pair $A, S$ is weakly compatible, we have $A S v=S A v$ and then $S S v=S A v=A S v=$ $A A v$.

Next we claim that $S A v=A v$. Putting $x=A v$ and $y=u$, we have

$$
\begin{aligned}
d(S A, T u)= & d(S A v, T u) \\
\leq & {[\phi(d(A A v, B u), d(A A v, S A v),} \\
& d(B u, T u), d(S A v, B u), d(A A v v, T u))]^{\lambda} \\
\leq & d^{\lambda}(S A v, A v),
\end{aligned}
$$

which implies that $S A v=A v$ and hence $S A v=A v=A A v$. Hence $A v$ is common fixed point of $A$ and $S$. 
Also, one can easily prove that $B B u=B u=T B u$, that is, $B u$ is common fixed point of $B$ and $T$. As $A v=B u, A v$ is a common fixed point of $A, B, S$ and $T$.

Similarly we can complete the proof for cases in which $A X$, or $T X$, or $S X$ is a closed subset of $X$.

The uniqueness follows easily from inequality $(C 2)$. This completes the proof.

Finally, we prove the following theorems for weakly compatible mappings with common limit range property satisfying the implicit function in a multiplicative metric space.

Lemma 2.9. Let $A, B, S$ and $T$ be mappings of a multiplicative metric space $(X, d)$ satisfying the conditions $(C 1),(C 2)$ and the following condition:

$(C 7)$ the pair $A, S$ satisfies $C L R_{A}$ property or the pair $B, T$ satisfies $C L R_{B}$ property.

Then the pairs $A, S$ and $B, T$ share the common limit in the range of $A$ property or $B$ property.

Proof. Suppose that the pair $A, S$ satisfies common limit range of $A$ property. Then there exists a sequence $\left\{x_{n}\right\}$ in $X$ such that $\lim _{n \rightarrow \infty} S x_{n}=\lim _{n \rightarrow \infty} A x_{n}=$ $A z$ for some $z \in X$. Since $S X \subset B X$, so for each $x_{n}$ there exists $y_{n}$ in $X$ such that $S x_{n}=B y_{n}$. Then $\lim _{n \rightarrow \infty} B y_{n}=A z$. Hence, we have $\lim _{n \rightarrow \infty} S x_{n}=\lim _{n \rightarrow \infty} A x_{n}=$ $\lim _{n \rightarrow \infty} B y_{n}=A z$.

Now we claim that $\lim _{n \rightarrow \infty} T y_{n}=A z$. Putting $x=x_{n}$ and $y=y_{n}$ in $(C 2)$, we have

$$
\begin{aligned}
d\left(S x_{n}, T y_{n}\right) \leq[ & \phi\left(d\left(A x_{n}, B y_{n}\right), d\left(A x_{n}, S x_{n}\right)\right. \\
& \left.\left.d\left(B y_{n}, T y_{n}\right), d\left(S x_{n}, B y_{n}\right), d\left(A x_{n}, T y_{n}\right)\right)\right]^{\lambda}
\end{aligned}
$$

Letting $n \rightarrow \infty$, we have

$$
\begin{aligned}
d\left(A z, T y_{n}\right) & \leq\left[\phi\left(1,1, d\left(A z, T y_{n}\right), 1, d\left(A z, T y_{n}\right)\right)\right]^{\lambda} \\
& =d^{\lambda}\left(A z, T y_{n}\right)
\end{aligned}
$$

which implies that $\lim _{n \rightarrow \infty} T y_{n}=A z$.

Then the pairs $A, S$ and $B, T$ share the common limit in the range of $A$ property.

Similarly we can complete the proof for cases in which the pair $B, T$ satisfies common limit in the range of $B$ property. This completes the proof. 
Theorem 2.10. Let $A, B, S$ and $T$ be mappings of a multiplicative metric space $(X, d)$ satisfying the conditions $(C 1),(C 2)$ and $(C 7)$.

Then the pairs $A, S$ and $B, T$ have a coincidence point.

Moreover, assume that the pairs $A, S$ and $B, T$ are weakly compatible. Then $A, B, S$ and $T$ have a unique common fixed point.

Proof. From Lemma 2.9, the pairs $A, S$ and $B, T$ share the common limit in the range of $A$ property, that is, there exist two sequences $\left\{x_{n}\right\}$ and $\left\{y_{n}\right\}$ in $X$ such that

$$
\lim _{n \rightarrow \infty} S x_{n}=\lim _{n \rightarrow \infty} A x_{n}=\lim _{n \rightarrow \infty} B y_{n}=\lim _{n \rightarrow \infty} T y_{n}=A v
$$

for some $v \in X$.

Firstly, we claim that $A v=S v$. Putting $x=v$ and $y=y_{n}$ in $(C 2)$, we have

$$
\begin{aligned}
d\left(S v, T y_{n}\right) \leq[ & \phi\left(d\left(A v, B y_{n}\right), d(A v, S v),\right. \\
& \left.\left.d\left(B y_{n}, T y_{n}\right), d\left(S v, B y_{n}\right), d\left(A v, T y_{n}\right)\right)\right]^{\lambda} .
\end{aligned}
$$

Letting $n \rightarrow \infty$, we have

$$
\begin{aligned}
d(A v, A v) & \leq[\phi(1, d(A v, S v), 1, d(S v, A v), 1)]^{\lambda} \\
& \leq d^{\lambda}(A v, S v)
\end{aligned}
$$

which implies that $S v=A v$. Since $S X \subset B X$, there exists $w \in X$ such that $B w=S v$.

Now we claim that $B w=T w$. Putting $x=v$ and $y=w$, we have

$$
\begin{aligned}
d(B w, T w)= & d(S v, T w) \\
\leq & {[\phi(d(A v, B w), d(A v, S v),} \\
& d(B w, T w), d(S v, B w), d(A v, T w))]^{\lambda} \\
= & {[\phi(1,1, d(B w, T w), 1, d(B w, T w))]^{\lambda} } \\
= & d^{\lambda}(B w, T w),
\end{aligned}
$$

this implies that $B w=T w$ and hence $T w=B w=A v=S v$. Since the pairs $A, S$ and $B, T$ are weakly compatible and $A v=S v$ and $T w=B w$. Hence

$$
A S v=S A v=A A v=S S v, \quad T B w=B T w=B B w=T T w .
$$


Finally, we claim that $S A v=A v$. Putting $x=A v$ and $y=w$, we have

$$
\begin{aligned}
d(S A v, A v)= & d(S A v, T w) \\
\leq & {[\phi(d(A A v, B w), d(A A v, S A v),} \\
& \quad d(B w, T w), d(S A v, B w), d(A A v, T w))]^{\lambda} \\
\leq & d^{\lambda}(S A v, A v),
\end{aligned}
$$

this implies that $S A v=A v$ and hence $S A v=A v=A A v$, which implies that $A v$ is a common fixed point of $A$ and $S$.

Also, one can easily prove that $B B w=B w=T B w$, that is, $B w$ is a common fixed point of $B$ and $T$. As $A v=B w, A v$ is common fixed point of $A, B, S$ and $T$.

The uniqueness follows easily from $(C 2)$. This completes the proof.

\section{References}

[1] M. Aamri, D. El Moutawakil, Some new common fixed point theorems under strict contractive conditions, J. Math. Anal. Appl., 270 (2002), 181188. doi: 10.1016/S0022-247X(02)00059-8

[2] I. Altun, D. Türkoğlu, Some fixed point theorems for weakly compatible multivalued mappings satisfying an implicit relations, Filomat, 22 (2008), 13-21.

[3] I. Altun, D. Türkoğlu, Some fixed point theorems for weakly compatible mappings satisfying an implicit relations, Taiwanese J. Math., 13 (2009), 1291-1304.

[4] A.E. Bashirov, E.M. Kurplnara, A. Ozyapici, Multiplicative calculus and its applications, J. Math. Anal. Appl., 337 (2008), 36-48. doi: 10.1016/j.jmaa.2007.03.081

[5] F. Gu, L.M. Cui and Y.H. Wu, Some fixed point theorems for new contractive type mappings, J. Qiqihar Univ., 19 (2013), 85-89.

[6] X. He, M. Song, D. Chen, Common fixed points for weak commutative mappings on a multiplicative metric space, Fixed Point Theory Appl., 48 (2014), 9 pages. doi: 10.1186/1687-1812-2014-48

[7] M. Imdad, S. Kumar, M. S. Khan, Remarks on some fixed point theorems satisfying an implicit relations, Rad. Mat., 11 (2002), 135-143. 
[8] M. Imdad, B.D. Pant and S. Chauhan, Fixed point theorems in menger spaces using the $\left(C L R_{S T}\right)$ property and applications, J. Nonlinear Anal. Optim., 3 (2012), 225-237.

[9] G. Jungck, Common fixed points for noncontinuous nonself maps on nonmetric spaces, Far East J. Math. Sci., 4 (1996), 199-215.

[10] G. Jungck, B.E. Rhoades, Fixed points for set valued functions without continuity, Indian J. Pure Appl. Math., 29 (1998), 227-238.

[11] M . Özavsar, A.C. Çevikel, Fixed points of multiplicative contraction mappings on multiplicative metric spaces, arXiv:1205.5131v1 [math.GM], 2012.

[12] V. Popa, A fixed point theorem for mappings in $d$-complete topological spaces, Math. Moravica, 3 (1999), 43-48.

[13] V. Popa, A general coincidence theorem for compatible multivalued mappings satisfying an implicit relation, Demontsratio Math., 33 (2000), 159164.

[14] V. Popa, M. Mocanu, Altering distance and common fixed points under implicit relations, Hacet. J. Math. Stat., 38 (2009), 329-337.

[15] M. Sarwar, R. Badshah-e, Some unique fixed point theorems in multiplicative metric space, arXiv:1410.3384v2 [math.GM], 2014.

[16] S. Sharma, B. Deshpande, On compatible mappings satisfying an implicit relation in common fixed point consideration, Tamkang J. Math., 33 (2002), 245-252.

[17] W. Sintunavarat, P. Kumam, Common fixed point theorems for a pair of weakly compatible mappings in fuzzy metric spaces, J. Appl. Math., 2011 (2011), Article ID 637958, 14 pages. doi: 10.1155/2011/637958

[18] D. Türkoğlu, I. Altun, A common fixed point theorem for weakly compatible mappings in symmetric spaces satisfying an implicit relation, Bol. Soc. Mat. Mexicana, 13 (2007), 195-205. 
